\title{
Detecting the Triple Resistance Fault in Twisted Pair Telephone Lines
}

\author{
Islam Amin Ellabban \\ "New Damietta" Exchange- \\ Telecom Egypt \\ Central zone, New Damietta, \\ Damietta \\ 34517, Egypt
}

\author{
Mohamed Abouelatta \\ Faculty of Engineering-Ain \\ Shams University-Elec. \& \\ Comm. Eng. Dept. \\ 1-El-Sarayat St., Abbassia, \\ Cairo, 11517, Egypt
}

\author{
Abdelhalim Zekry \\ Faculty of Engineering-Ain \\ Shams University-Elec. \& \\ Comm. Eng. Dept. \\ 1-El-Sarayat St., Abbassia, \\ Cairo, 11517, Egypt
}

\begin{abstract}
Telecom Egypt Company (TE) is the unique copper fixed line network operator in Egypt and its primary aim is to improve the efficiency of this network to face the increasing demand for high data rates communications. The severe fault affects the copper network is the resistance fault. The most commonly used testing and measuring instrument in TE is "Dynatel 965DSP" which has some drawbacks.
\end{abstract}

This paper introduces a new methodology for the determination of Resistance Fault locations of a three resistance faults twisted pair telephone line. This method is based on the three voltmeter method and automated by using NI USB-6281 Data Acquisition Card (DAQ). The simulation results accuracy of one of the faults does not exceed $1.63 \%$ for faults up to $100 \mathrm{~K} \Omega$ and $350 \mathrm{~m}$ length cable.

\section{General Terms}

A new methodology for determination of resistance fault locations, three voltmeter method, Data Acquisition System

\section{Keywords}

Telecom Egypt Company; Dynatel 965DSP; Three voltmeter method; Resistance Fault Location (RFL); Data Acquisition Card

\section{INTRODUCTION}

The demand on high data rates across the telephone copper network in Egypt increases dramatically. The public telephone network in Egypt must be of high performance to satisfy the requirements of this demand. To improve the network performance, the telephone cable faults have to be accurately determined and repaired to achieve this target. The new trend in TE is to replace the primary copper network with fiber cables without replacing the secondary copper network $[1,2]$. As the secondary network is susceptible to different types of faults, the determination of ground resistance faults locations represents the real challenge to engineers and technicians [37]. In TE, "Dynatel 965DSP" is the most commonly used instrument to determine such faults. This instrument is expensive in addition, it cannot measure some types of resistance faults; low insulation resistance for both wires of a telephone line with ground.
In this work, a new reliable, cheap and sufficiently accurate automatic measurement method used in measuring the telephone line electrical parameters with sufficient accuracy is introduced to determine the fault locations of a three resistance faults twisted pair telephone line [8]. This method is based on the adaptive three voltmeter method [9-11] with NI USB-6281 Data Acquisition Card (DAQ) [12-14] and a Personal Computer (PC).

The paper is organized as follows: section 2 contains the three voltmeter method principle; section 3 introduces RFL using the three voltmeter method including the simulation results for different cases; section 4 shows the experimental results. Finally, the conclusions are presented.

\section{THREE VOLTMETER METHOD PRINCIPLE}

The three voltmeter method is primarily suited for power and energy measurements, but it is also well suited for impedance measurements provided that suitable reference impedance and three highly-accurate voltmeters are available [9-11]. Recently, the three voltmeter method has been found very convenient for routine calibration of standards of selfinductance, inductor losses, and it has also been applied to calibration of the mutual inductance. The basic arrangement of the measurement method is shown in Figure 1, where $Z_{x}=R_{x}+j X_{x}$ is the Device Under Test (DUT) and $Z_{s}=$ $R_{S}+j X_{S}$ is the reference impedance standard. Referring to [9$11]$, the three voltmeter method is explained in details.

In our work, the RFL determination depends on the measurement of the wire DC resistance, and thus the unknown impedance $Z_{x}$ will be of resistive part only; $R_{x}$. The voltages $V_{x}, V_{s}$ and $V_{T}$ can be measured across $R_{x}, R_{s}$ and the DC signal source respectively. In this case, the value of the unknown resistance is calculated using equation (1).

$R_{x}=\frac{V_{x}}{V_{s}} R_{s}$

Figure 2 illustrates the RFL measurement circuit. There is no need for the peak detectors illustrated in Figure 1. 


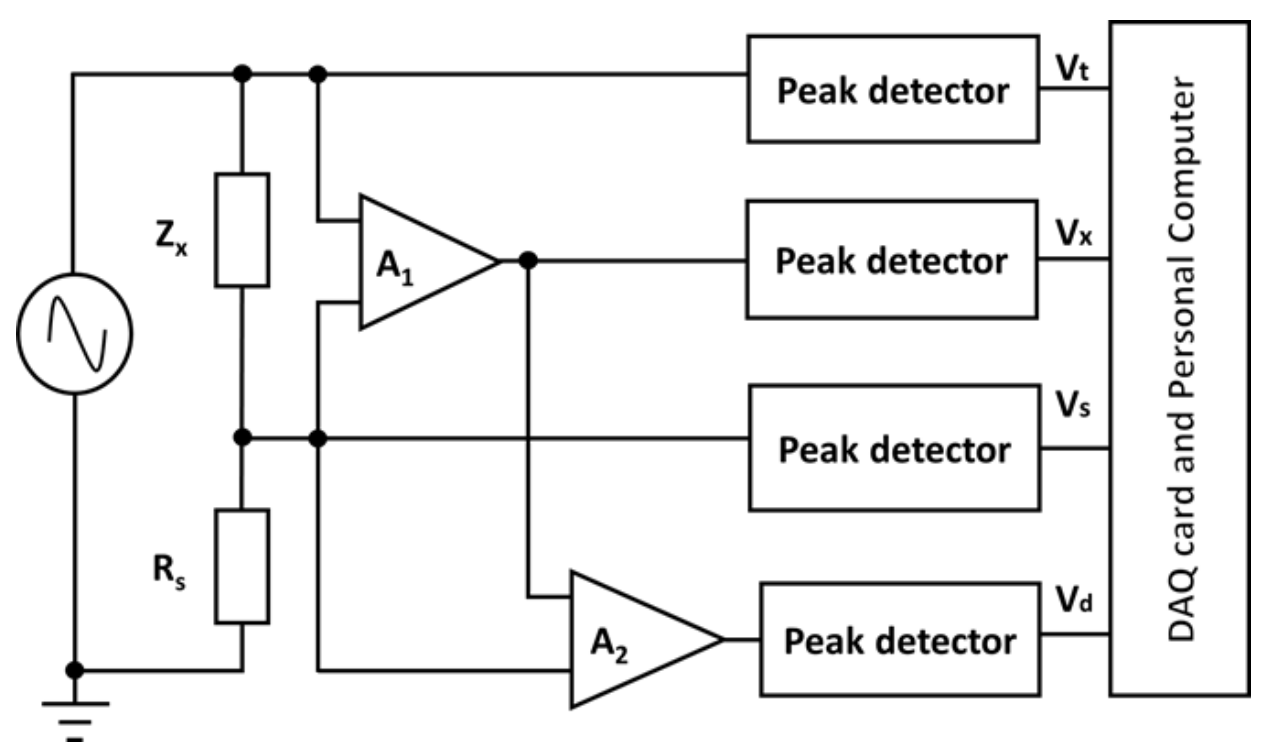

Fig 1: The modified three voltmeter method measuring circuit

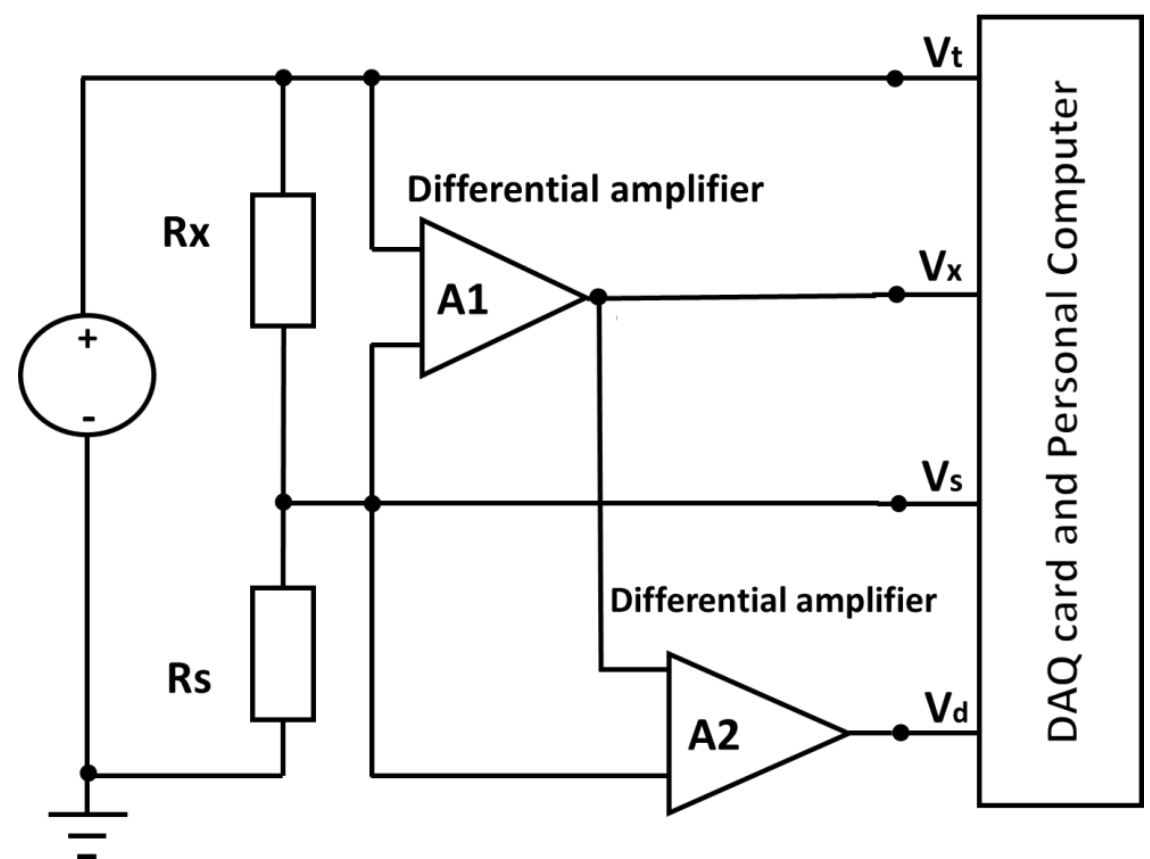

Fig 2: Reduced three voltmeter method RFL measuring circuit

\section{RFL OF A THREE RESISTANCE FAULTS TELEPHONE LINE}

The RFL test purpose is to determine the location of the fault (usually a splice) from both near and far ends with the highest accuracy. The engineer should check the continuity of the line, and then the insulation resistance measurement of the two conductors with the ground have to be performed. Assuming that we have a transmission line that is continuous along its path and has three resistance faults as shown in Figure 3.

In the Figure, $A$ and $B$ are the near end terminals and $C$ and $D$ are the far end terminals. The two terminals $A$ and ground are connected in the circuit as the unknown resistance while the two terminals $C$ and $D$ are still open. The measured unknown resistance $R_{e}$ is described by equation (2) 


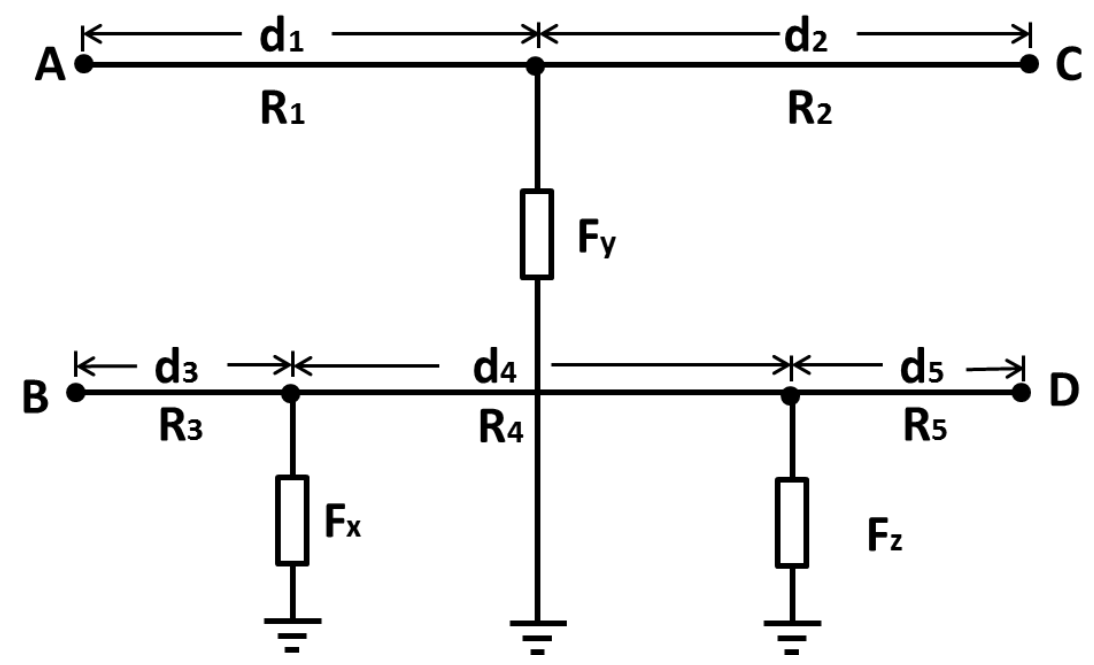

Fig 3: Three resistance faults telephone line

$R_{e}=R_{1}+F_{y}+0.211 d_{1}$

Where $R_{l}$ is the wire DC resistance from terminal $A$ to the location of the fault $F_{y}$ and $d_{l}$ is the distance between terminal $A$ and the fault $F_{y}$. The DC resistance of the ground wire is $0.211 \Omega / \mathrm{m}$.

Applying the above mentioned sequence for terminals $A$ and ground as the unknown resistance when the two terminals $C$ and $D$ are strapped (S.C.). By neglecting the value of the copper and the ground wires resistances with respect to the faults values, the measured unknown resistance $R_{f}$ is described by equation (3)

$R_{f} \cong R_{1}+\left(\frac{F_{x} F_{y} F_{z}}{F_{x} F_{y}+F_{y} F_{z}+F_{x} F_{z}}\right)+0.211 d_{3}$

Where $F_{x}$ and $F_{z}$ are two ground faults. $d_{3}$ is distance between terminal $B$ and the fault $F_{x} . R_{3}$ is wire the DC resistance from terminal $B$ to the fault $F_{x}$.

By connecting $B$ and ground as the unknown resistance and neglecting the value of $R_{4}$ and the ground wire resistance with respect to the faults values, the measured unknown resistance $R_{k}$ is described by equation (4)

$R_{k} \cong R_{3}+\left(\frac{F_{x} F_{z}}{F_{x}+F_{z}}\right)+0.211 d_{3}$

By using terminals $C$ and ground as the unknown resistance, the measured unknown resistance $R_{c}$ is described by equation (5)

$R_{c}=R_{2}+F_{y}+0.211 d_{2}$

Where $R_{2}$ is the wire DC resistance from terminal $C$ to the fault $F_{y}$ and $d_{2}$ is the distance from terminal $C$ to the fault $F_{y}$.

If the two terminals $C$ and ground are used to represent the unknown resistance terminals when terminals $A$ and $B$ are strapped, the measured unknown resistance is $R_{d}$. By neglecting the value of the copper and ground wires resistances with respect to the faults values, $R_{d}$ is described by equation (6).

$R_{d} \cong R_{2}+\left(\frac{F_{x} F_{y} F_{z}}{F_{x} F_{y}+F_{y} F_{z}+F_{x} F_{z}}\right)+0.211 d_{5}$
Where $d_{5}$ is the distance from terminal $D$ to the fault $F_{z}$. The wire DC resistance from terminal $D$ to the fault $F_{z}$ is $R_{5}$.

Finally, repeating the previous sequence for terminals $D$ and ground and neglecting the value of $R_{4}$ and the ground wire resistance with respect to the faults values, the measured unknown resistance $R_{j}$ is described by equation (7)

$R_{j} \cong R_{5}+\left(\frac{F_{x} F_{z}}{F_{x}+F_{z}}\right)+0.211 d_{5}$

Equations (2) to (7) are solved simultaneously to determine the fault locations and the fault resistance $F_{y}$.

\subsection{Three Resistance Faults Telephone Line Simulation Results}

Assuming a twisted pair of three resistance faults of length $350 \mathrm{~m}$ is simulated. The fault $F_{y}$ is $100 \mathrm{~m}$ away from the near end terminal $A, d_{l}$ and $250 \mathrm{~m}$ away from the far end terminal $C, d_{2}$. Also, assuming that the fault $F_{x}$ is $50 \mathrm{~m}$ away from the near end terminal $B, d_{3}$ and the fault $F_{z}$ is $180 \mathrm{~m}$ away from the far end terminal $D, d_{5}$. The distance between the faults $F_{x}$ and $F_{z}, d_{4}$ is $120 \mathrm{~m}$. Referring to [12-14], NI USB-6281 DAQ card specifications are used in the simulation process. The simulation figures illustrate the error percentage of fault locations for fault resistances starting from $1 \mathrm{~K} \Omega$ up to 100 $\mathrm{K} \Omega$ using a DC voltage source of $10 \mathrm{~V}$ taking into account the DAQ card absolute accuracy and resolution in DC measurement processes. The faults values are considered equal at every simulation process. The distances errors are illustrated for different cases of reference (known) resistances. Figures 4, 5, 6, 7 and 8 demonstrates the error in distances $d_{l}$, $d_{2}, d_{3}, d_{4}$ and $d_{5}$ respectively using adaptive resistance $R_{s}$ as the known resistance in the case of 18 bit resolution (5.5 digits) DAQ card (NI USB-6281) and 16 bit resolution (4.5 digits) DAQ card (NI USB-6210). Adaptive resistance measurement depends on using a potentiometer to change the known (variable) resistance value until reaching the point at which both $V_{x}$ and $V_{s}$ are nearly equal. This is because the three voltmeter method exhibits the best accuracy when both the known and unknown resistances are nearly equal. 


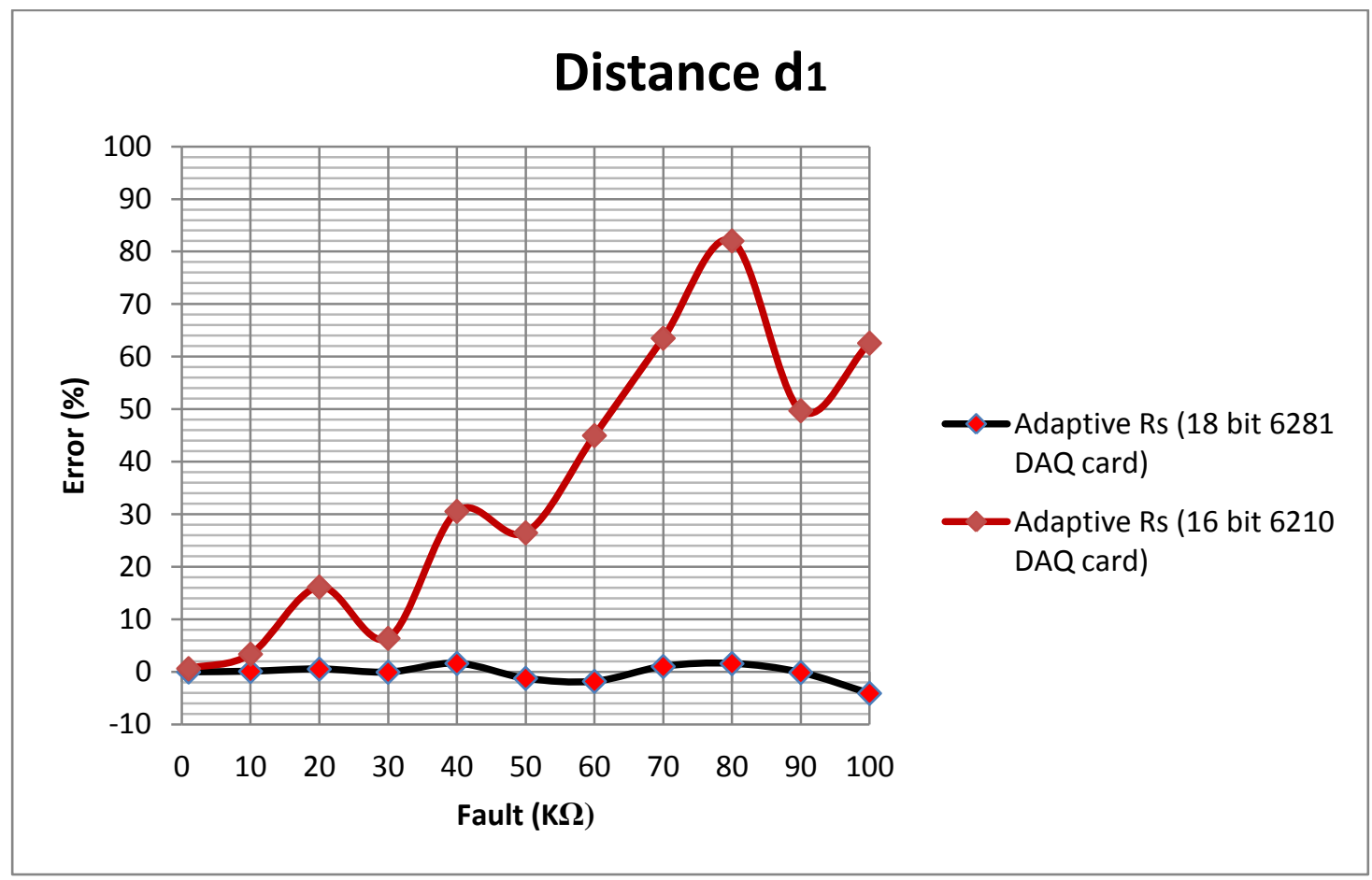

Fig 4: Distance $d_{1}$ simulation error at different known resistance values

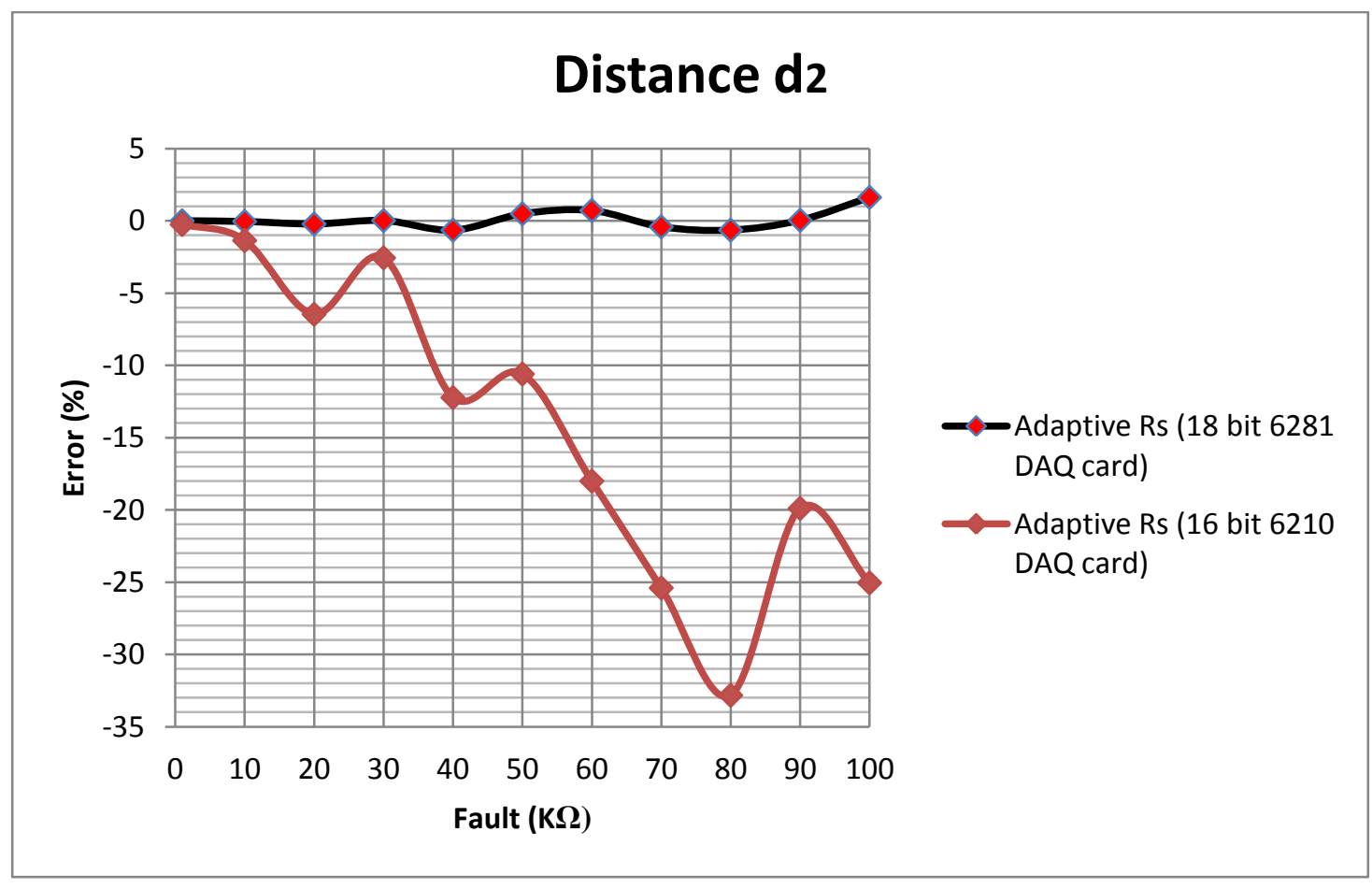

Fig 5: Distance $d_{2}$ simulation error at different known resistance values 


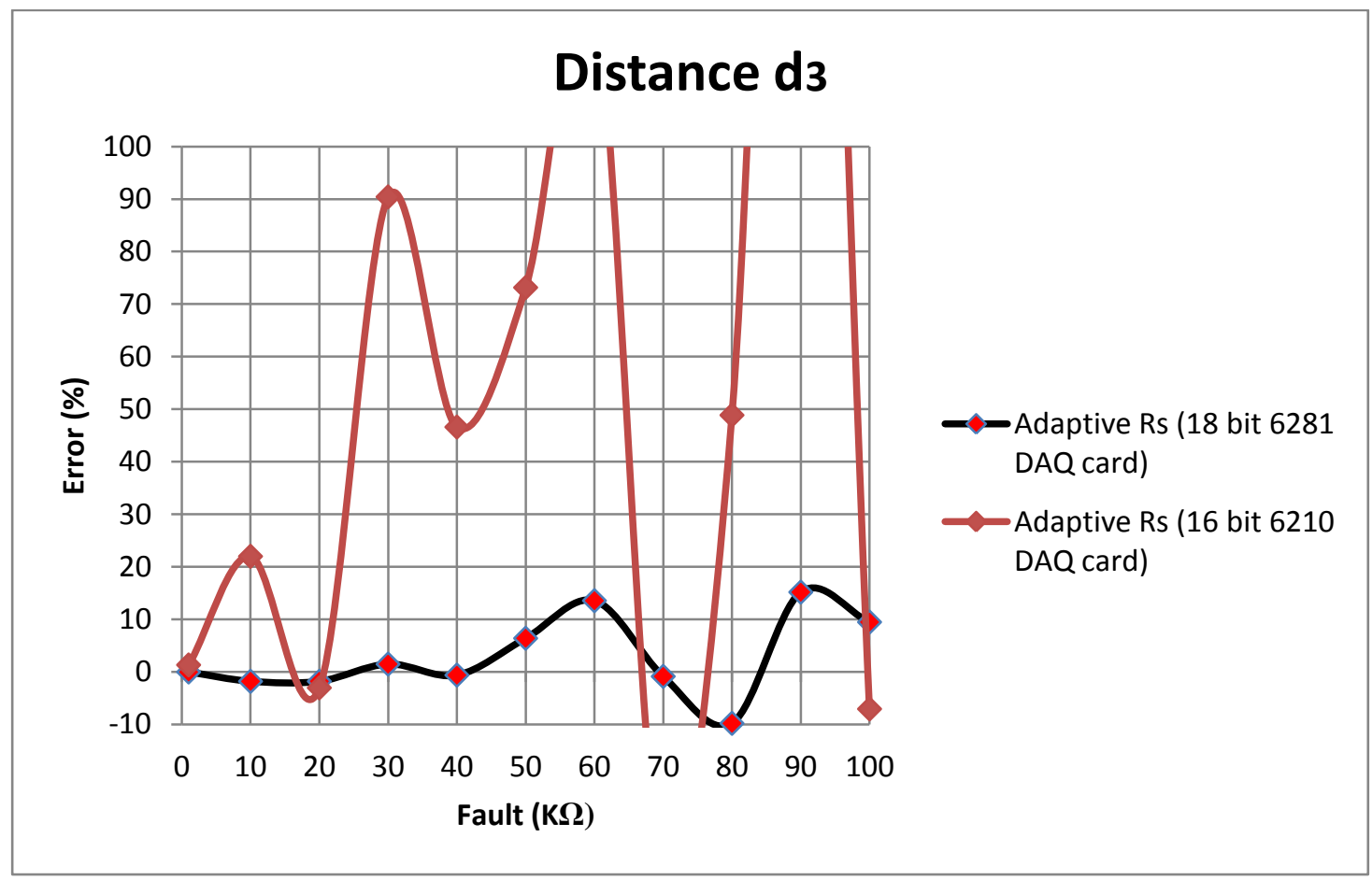

Fig 6: Distance $d_{3}$ simulation error at different known resistance values

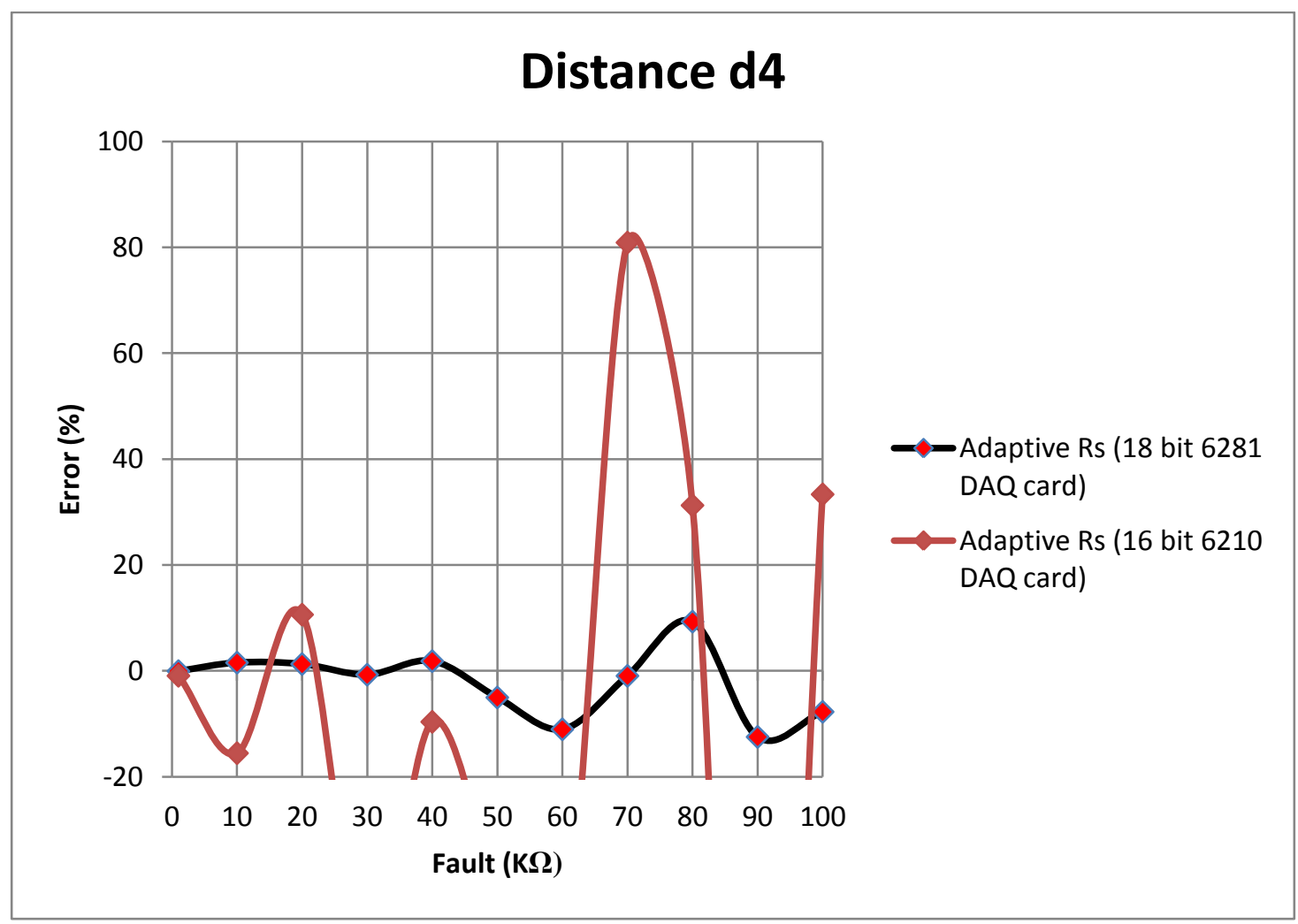

Fig 7: Distance $d_{4}$ simulation error at different known resistance values 


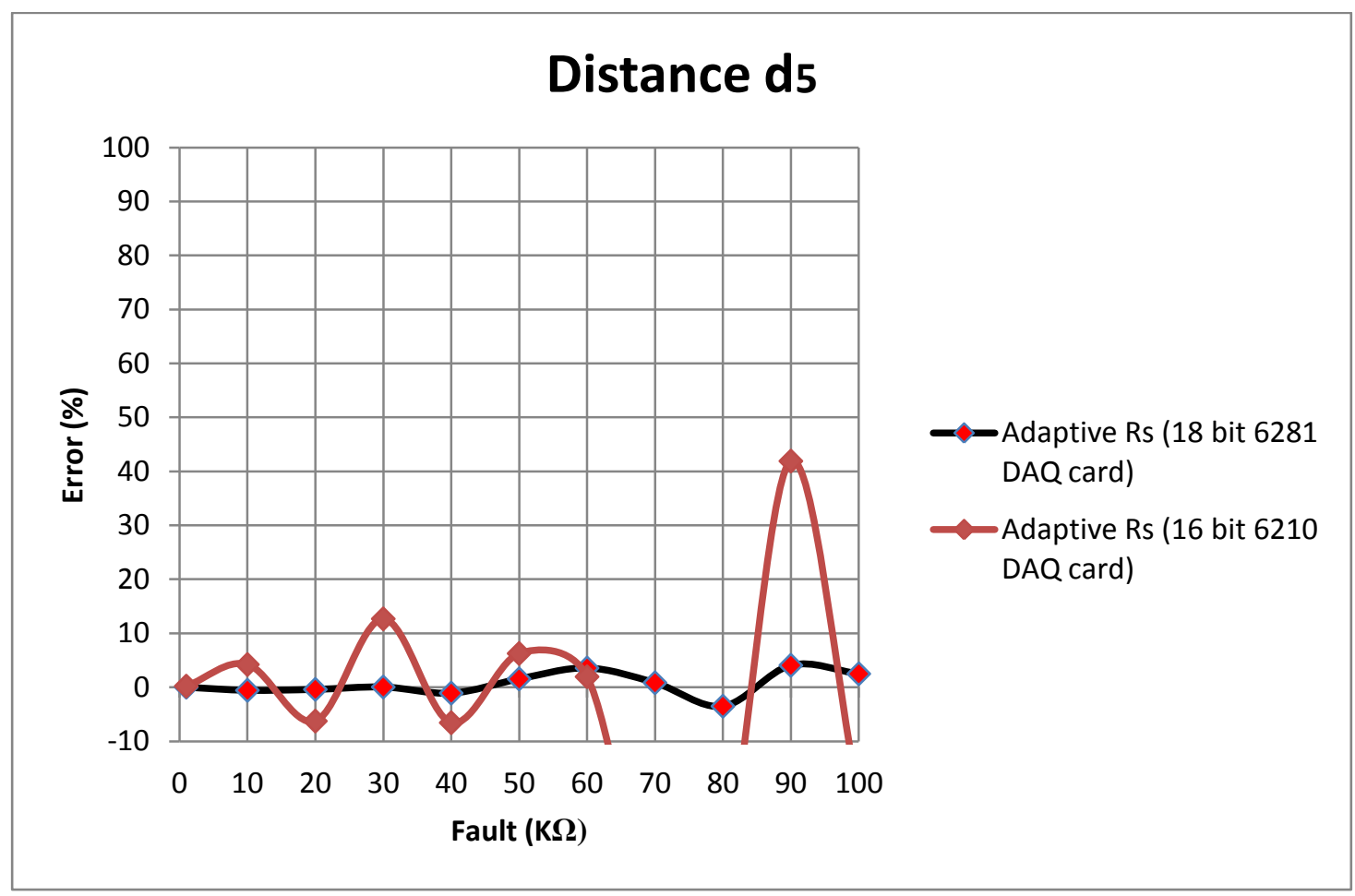

Fig 8: Distance $d_{5}$ simulation error at different known resistance values

\subsection{Analysis of Simulation Results}

The simulation distance is chosen to be $350 \mathrm{~m}$ which is the average length of the secondary telephone cable of MultiService Access Node (MSAN) new cabinet in TE. The simulation process was performed using adaptive resistance $R_{s}$ as the known resistance and for two cases of DAQ resolution; 18 bit and 16 bit resolution DAQ card. The concept of adaptive resistance is illustrated before. In our simulation, it is assumed that $F_{x}, F_{y}$ and $F_{z}$ have equal values. The simulation results for distance $d_{l}$ error show that they have acceptable accuracy ( $<10 \%$ error) in the case of 18 bit resolution DAQ card for all values of faults. The results obtained 16 bit resolution DAQ card have acceptable accuracy for faults up to $30 \mathrm{~K} \Omega$ except at value of $20 \mathrm{~K} \Omega$. For faults higher than 30 $\mathrm{K} \Omega$, the accuracy is not acceptable.

For the distance $d_{2}$, the simulation results using 18 bit resolution DAQ card (NI USB-6281) have acceptable accuracy for all values of faults and does not exceed $1.63 \%$ for all fault values. The results obtained using adaptive known resistance in the case of 16 bit resolution DAQ card have acceptable accuracy for faults up to $30 \mathrm{~K} \Omega$ and suffer from large error values for faults greater than this value.

For distance $d_{3}$ simulation error, the results obtained using adaptive known resistance in the case of 18 bit resolution DAQ card (NI USB-6281) are not acceptable for fault values of $60 \mathrm{~K} \Omega$ and $90 \mathrm{~K} \Omega$. For other fault values, the results are acceptable. The results obtained using adaptive known resistance in the case of 16 bit resolution DAQ card have acceptable accuracy for faults of values $1 \mathrm{~K} \Omega, 20 \mathrm{~K} \Omega$ and 100 $\mathrm{K} \Omega$ only.

For distance $d_{5}$ simulation error, the results obtained using adaptive known resistance in the case of 18 bit resolution DAQ card (NI USB-6281) are acceptable for all fault values. The results obtained using adaptive known resistance in the case of 16 bit resolution DAQ card have acceptable accuracy for fault values up to $60 \mathrm{~K} \Omega$ except at the value of $30 \mathrm{~K} \Omega$. For faults greater than $60 \mathrm{~K} \Omega$, the accuracy is not acceptable.

Distance $d_{4}$ is calculated using equation (10)

$d_{4}=d-d_{3}-d_{5}$

Where $d$ is the twisted pair telephone line length starting from the near end to the far end terminals. The error in $d_{4}$ is due to the errors in $d_{3}$ and $d_{5}$ as illustrated in equation (8).

The accuracy of measurement using the three voltmeter method is improved when the value of both the known and unknown resistances are nearly equal. From the previous discussion, it is noticeable that the results using 18 bit resolution DAQ card are better than those obtained using 16 bit resolution DAQ card. In spite of using adaptive resistance, equations (2) to (7) show that the faults values are greater than the copper wire and ground wire resistances which yields to measurements errors. Equations (3), (4), (6) and (7) are based on the assumption that the copper and ground wire resistances are negligible with respect to the faults values in determination of parallel resistances equivalent value which affects the results accuracy.

Another reason for the obtained error is the absolute accuracy of the used DAQ card as it is not negligible because the voltages across the DC wires resistances are very small. Thus, adding it to the actual voltages causes fault location error. The absolute accuracy of 18 bit DAQ card is better than the absolute accuracy of 16 bit DAQ card.

\section{EXPERIMENTAL RESULTS}

The keyword of measurements accuracy is the DAQ card resolution; as the DAQ card resolution increases, the measurements accuracy increases. An experiment is performed using (NI USB-6008) low cost DAQ card on a single faulty wire jelly-filled cable of length $16.7 \mathrm{~m}$ and 
different fault resistance $F_{x}$ values at distance of $8.8 \mathrm{~m}$ from the near end port as shown in Figure 9. The used DAQ card in this experiment has a resolution of 12 bit. The adaptive $R_{S}$ results emphasizes that the accuracy of the results are acceptable for fault values less than or equal to $100 \Omega$. Table 1 illustrates the obtained experimental results.

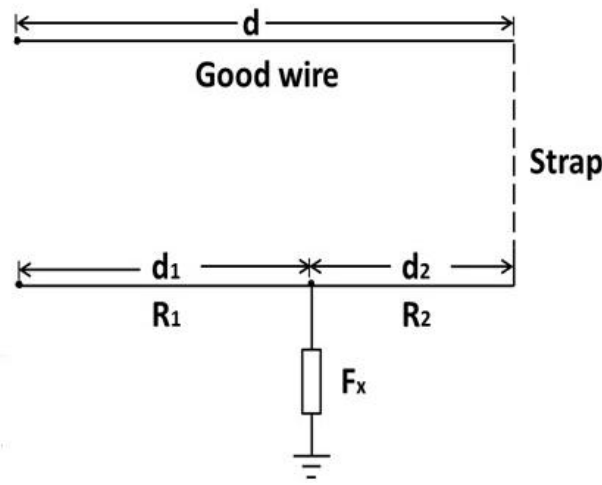

Fig 9: Single faulty wire telephone pair

As the reference resistance is nearly equal to the unknown resistance (the fault resistance plus the wire resistance), the measurement accuracy shows acceptable performance. More accurate results can be obtained in this case if the line length is longer than the length used in the experiment. The accuracy depends on the DAQ card resolution; as the DAQ card resolution increases, the result accuracy increases and vice versa.

Table 1. NI USB-6008 experimental results

\begin{tabular}{|c|c|c|}
\hline $\mathbf{F}_{\mathbf{x}}(\boldsymbol{\Omega})$ & $\mathbf{d}_{\mathbf{1}}$ (experiment) & Error $(\boldsymbol{\%})$ \\
\hline 12 & 9.259 & 5.21 \\
\hline 100 & 9.376 & 6.54 \\
\hline 1000 & 16.7 & 89.77 \\
\hline
\end{tabular}

\section{CONCLUSIONS}

The simulation results show that the error in distance $d_{l}$ does not exceed $-4.09 \%$ for all faults values using adaptive known resistance in the case of 18 bit DAQ card (NI USB-6281). In the case of 16 bit DAQ card using adaptive known resistance, the error does not exceed $6.37 \%$ for faults up to $30 \mathrm{~K} \Omega$ except at the value $20 \mathrm{~K} \Omega$ and is not acceptable for fault value greater than $30 \mathrm{~K} \Omega$. Distance $d_{2}$ location error has the same results as $d_{l}$ using adaptive known resistance and 18 bit DAQ card, the results accuracy is acceptable for all fault values and the error does not exceed $1.63 \%$. In the case of adaptive known resistance with 16 bit DAQ card, the results are acceptable for faults up to $30 \mathrm{~K} \Omega$ and does not exceed $6.46 \%$ error. For distance $d_{3}$, the results obtained using adaptive known resistance with 18 bit DAQ card are acceptable for all fault values and does not exceed $-9.8 \%$ except at the value of $60 \mathrm{~K} \Omega$ and $90 \mathrm{~K} \Omega$. In the case of 16 bit resolution DAQ card, the results accuracy is not acceptable for most values of the faults.

For distance $d_{5}$, the results obtained using adaptive known resistance with 18 bit DAQ card do not exceed $4.09 \%$ error for all fault values. The results obtained using adaptive known resistance with 16 bit DAQ card have acceptable accuracy for fault values up to $60 \mathrm{~K} \Omega$ and does not exceed $-6.54 \%$ error except at the value of $30 \mathrm{~K} \Omega$. For faults greater than $60 \mathrm{~K} \Omega$, the results accuracy is not acceptable.

The experimental results accuracy depends on the DAQ card resolution; the higher DAQ card resolution, the better results accuracy.

"Dynatel 965DSP" can measure only one resistance fault for one wire of the twisted pair line and the other wire must not have any faults. The measurement results using "Dynatel 965DSP" illustrate that the error is $7.16 \%$ or higher for different fault values.

The determination of resistance fault location using the three voltmeter method has many advantages. First, the three voltmeter circuit is simple and cheap. Second, many cases of resistance faults can be measured on the contrary to "Dynatel 965DSP". Third, the program is simple to be used by engineers and technicians. Finally, the fault locations can be determined easily.

The main disadvantage of the three voltmeter method is that its accuracy depends on the accuracy of the measured voltages and the used DAQ card.

As a future work, we will improve the resistance fault location by using the four voltmeter method in addition to using high accuracy DAQ card. Using numerical methods in solving the equations can yield to more accurate results.

\section{ACKNOWLEDGMENTS}

The article is partially supported by a grant of the Foundation of Computer Science, NY, USA vide FCS/RT56/15

Thanks to Telecom Egypt Company for the permission of using its technical data.

\section{REFERENCES}

[1] Gallon, C., Chohan, D., \& James, S. (2009). NextGeneration Access Architectures and Distributed MSAN Concept. FUJITSU Sci. Tech. J, 45(4), 339-345.

[2] Zhang, Y., \& Duysters, G. (2010). Alliance-based Network View on Chinese Firms' Catching-up: Case Study of Huawei Technologies Co. Ltd. Journal on Innovation and Sustainability. RISUS ISSN 2179$3565,1(2)$.

[3] Nishiyama, E., Nishijima, C., \& Kuwanami, K. (2004, November). A method for fault detecting on twisted pair cable network by use of m-sequence correlation. In Industrial Electronics Society, 2004. IECON 2004. 30th Annual Conference of IEEE (Vol. 2, pp. 19291934). IEEE.

[4] Neus, C., Foubert, W., Rolian, Y., Maes, J., \& Van Biesen, L. (2008). Feasibility and challenges of DSL loop make-up identification via single-ended line tests. Metrology and Measurement Systems, 15(4), 473484 .

[5] Shi, Q., Troeltzsch, U., \& Kanoun, O. (2010, June). Detection and localization of cable faults by time and frequency domain measurements. In Systems Signals and Devices (SSD), 2010 7th International Multi-Conference on (pp. 1-6). IEEE.

[6] Shi, Q., \& Kanoun, O. (2013, May). Automated wire fault location using impedance spectroscopy and differential evolution. In Instrumentation and 
Measurement Technology Conference (I2MTC), 2013 IEEE International (pp. 359-364). IEEE.

[7] Shi, Q., \& Kanoun, O. (2012, March). System simulation of network analysis for a lossy cable system. In Systems, Signals and Devices (SSD), 2012 9th International MultiConference on (pp. 1-5). IEEE.

[8] Ellabban, I. A., Abouelatta, M., \& Zekry, A. (2014). Measurement of Telephone Line Parameters using the Three Voltmeter Method. Measurement, 107(11).

[9] Muciek, A., \& Cabiati, F. R. A. N. C. O. (2006). Analysis of a three-voltmeter measurement method designed for low-frequency impedance comparisons.Metrology and Measurement Systems, 13(1), 19-33.
[10] Dumbrava, V., \& Svilainis, L. (2007). The automated complex impedance measurement system. Elektronika ir elektrotechnika, (4), 59-62.

[11] Tumanski, S. (2006). Principles of electrical measurement. CRC press.

[12] Tong, Y., Ye, G., Guo, K., \& Li, H. (2011). Highly accurate calibration system for electronic instrument transformers. Metrology and Measurement Systems, 18(2), 315-322.

[13] Ling, S. U. N. (2010). Design of high speed data acquisition system based on USB-6281. Electronic Design Engineering, 11, 068

[14] Zuch, E. L. (1979). Data Acquisition and Conversion Handbook. Datel-Intersil, Inc. 